\title{
Fields in Electrically Short Ground Systems: An Experimental Study
}

\author{
A. N. Smith ${ }^{1}$ and T. E. Devaney ${ }^{1}$
}

(April 20, 1959)

\begin{abstract}
An experimental study of magnetic field distribution in a simplified radial ground system on poorly conducting soil under an electrically short, toploaded monopole is described. It is shown that the distribution is that expected from the theory of $\mathrm{J}$. R. Wait in those portions of the radial system satisfying the assumptions of the theory, and that the theory may still be successfully applied for $H$-field power loss computations even when this is not fully the case. The particular model system studied exhibits a condition suggesting damped standing waves on the radials in the area where the radial spacing exceeds that required by the theory.
\end{abstract}

\section{Introduction}

For an electrically small monopole, the groundsystem power losses very often are a controlling factor in radiation efficiency. The decision to construct at Cutler, Maine, a new high-power, very-lowfrequency station with specified efficiency greatly exceeding that of any such existing station has thus resulted in a large amount of recent interest in ground-system design.

The final ground-system configuration for Cutler came basically from computations based on Wait's $[1,2,3]$ equivalent surface impedance formula for radial current ( $H$-field) power loss for a plane, radial grid of wires laid with perfect contact on an earthair interface; and from design modifications brought about according to ground charging current ( $E$-field) power loss computations based on formulas due to Abbott [4], Wheeler [5], and Wait [6]. The soundness of the theoretical design was partially and indirectly confirmed at the U.S. Navy Electronics Laboratory, San Diego, Calif., by comparing losses derived from impedance measurements [7] with those computed from theory for a model toploaded vlf monopole over a radial-wire system and also over a perfectly conducting ground [8]. A somewhat more direct test of theory was made by W. G. Hutton and others of Smith Electronics, Inc., Cleveland, Ohio $[9,10]$, who measured the field distribution (magnitude only) in a full-scale, fan-shaped ground-system sector constructed at the Cutler site. An analysis of his data by Wait [11] showed that the measured field distribution did indeed generally agree with that expected from a division of ground system current according to the surface impedance ratio derived from the theory.

However, there existed sufficient uncertainty about some conditions in all these experiments that a refined field-distribution study seemed desirable. Accordingly, a phase- and magnitude-measurement program was carried out on the vlf model previously used for impedance measurements. The dual objective was to test the validity of the theory and to investigats more adequately the direction and degree of departures therefrom those existing in the model.

\footnotetext{
${ }^{1}$ U.S. Navy Electronics Laboratory, San Diego, Calif.
}

\section{Theoretical Discussion}

The working formula for ground loss $R_{g}$ in terms of the azimuthal magnetic field $H_{\phi}(\rho, \theta)$ at the surface of the ground $(\theta=0)$ referred to the antenna feed point where current $I_{\mathrm{o}}$ flows is

$$
R_{g}=\text { Real part of } \frac{1}{I_{0}^{2}} \int_{r_{i}}^{\infty} Z\left[H_{\phi}(\rho, 0)\right]^{2} 2 \pi \rho d \rho .
$$

This equation is derived $[2,3]$ from the incremental Poynting vector flux due to the tangential component $\left(E_{\rho}\right)$ of electric field arising from the finite conductivity of the soil. In the derivation use is made of an approximate boundary condition through which the flux is expressed in terms finally of $H_{\phi}$, the magnetic field of the monopole over an infinitely conducting ground. For convenience the integration is performed over two regions, $r_{i} \leq \rho \leq r$, and $\rho \geq r, r$ being the length of the wire radials, since, in the area occupied by the ground system, the surface impedance $Z$ is the combination of wire impedance $Z_{w}$ and soil impedance $Z_{s}$ and in the remainder it is simply $Z_{s} . \quad r_{i}$ is the inner extremity of the radials

The five major assumptions made in deriving equa tion (1) are stated below.

(1) The approximate boundary condition $E_{\rho}=Z H_{\phi}$ is used, requiring that $\rho H$ change slowly in a distance $d=\left|\gamma^{-1}\right|$ where $\gamma=\left[i \mu \omega\left(\sigma+i \omega k \epsilon_{o}\right)\right]^{1 / 2}$ is the propagation constant of the soil.

(2) The combined surface impedance varies slowly in $\left|\gamma^{-1}\right|$

(3) The radial wire screen of $N$ radials in any small region a distance $\rho$ from the antenna base equals that of a grid of parallel wires of the same mean spacing $s=2 \pi \rho / N$, i.e., $Z_{w}=\left(i \eta_{o} s / \lambda\right) \ln (s / 2 \eta c)$ where $\eta_{o}=377 \mathrm{ohms}$, and $c$ is the wire cross section radius. This requires the condition $s \ll\left|\gamma_{e}{ }^{-1}\right|$ where $\gamma_{e}=\left(\frac{\gamma_{0}^{2}+\gamma^{2}}{2}\right)^{1 / 2}$ is the effective propagation constant of the composite medium at the interface $\left(\gamma_{o}=i \beta\right)$.

(4) To replace the actual magnetic field $H_{\phi}$ by $H_{\phi}^{\infty}$ at the surface of the ground it is necessary that 
$\left|\gamma^{-1}\right| \ll 1 / \beta$, i.e., the soil skin depth be much less than a free-space electrical radian.

(5) The surface impedances of screen and soil must combine as simple parallel impedances, i.e., mutual coupling and potential differences between wires and soil are zero, so that

$$
Z=Z_{s} Z_{w} /\left(Z_{s}+Z_{w}\right)
$$

within the ground system.

These assumptions must be satisfied in setting up any direct experimental test of equation (1).

Since this paper is largely concerned with the field structure due to division of current in the ground system according to assumption (5), its implications are now developed. The total ring current crossing a cylindrical boundary of radius $\rho$ drawn about the monopole base as a center is $I_{t}=I_{w}+I_{s}=2 \pi \rho H_{\phi}$. Normalizing with respect to base current $\left(h_{\phi}=H_{\phi} / I_{o}\right)$ and removing the factor $2 \pi \rho$, assumption (5) leads to

$$
h_{\phi}=h_{\phi} Z_{s} /\left(Z_{w}+Z_{s}\right)+h_{\phi} Z_{w} /\left(Z_{w}+Z_{s}\right) .
$$

The first term on the right of (3) corresponds to wire current $I_{w}$. Letting $I_{w} / I_{0}=N i_{w}$, so that $i_{w}$ is the normalized current in one radial wire, the $I_{w}$ term is seen to be exactly $N i_{w} / 2 \pi \rho$. Hence the magnetic field $h_{w}$ at a field point $d$ meters from the wire center is

$$
h_{w}=h_{\phi} \rho Z_{s} / N d\left(Z_{w}+Z_{s}\right) .
$$

The second term on the right of (3) corresponds to current in the soil $I_{s}$, and in fact is $i_{s} / 2 \pi \rho$, where $i_{s}=I_{s} / I_{0}$. If the wire centers lie exactly in the plane of the earth-air interface, then the magnetic field tangent to it, $h_{s}$, can be simply evaluated by applying Ampere's law to the path illustrated in figure 1, where $n$, the number of skin depths $\delta$ from the surface to the portion def of the path, is so large that $H_{\phi}$ along it is negligibly small. At the interface there is no tangential contribution from the wires themselves, and along the vertical portion the contributions cancel. Hence

$$
2 \pi \rho h_{s}=N i_{w} / 2+i_{s}
$$

Subtracting this from (3), it is seen that

$$
h_{s}=\frac{h_{\phi}}{2}\left(1+\frac{Z_{w}}{Z_{s}+Z_{w}}\right) .
$$

That is, in the plane of the wire centers the total tangential field is the incident field of the downlead plus a contribution due to the induced current flowing in the soil. The wires carry only a portion of the image current. In the special case of a wire counterpoise and no soil, the second term vanishes, and all the image current flows in the wires. In the case of infinitely conducting ground, on the other hand, the second term also equals $h_{\phi} / 2$ (perfect reflection), the

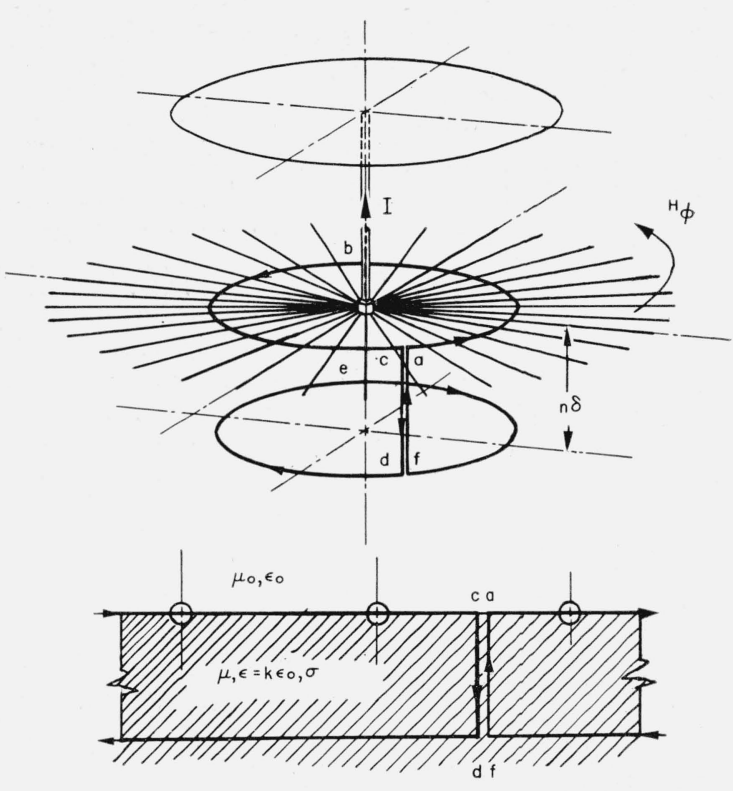

FiguRE 1. Integration path for evaluating $\mathrm{h}_{s}$.

distinction between $h_{w}$ and $h_{s}$ is lost, and the total field is just $h_{\phi}$.

From the above considerations one sees that to determine the validity of the theory of $H$-field power losses, it is sufficient to observe that the fields $h_{w}$ and $h_{s}$ satisfy equations (4) and (6) when assumptions (1), (2), (3) and (4) are met.

\section{Experimental Procedure}

In the Cutler design [11] and in the NEL models used for the impedance measurements, soil constants and ground system parameters were such that assumptions (1), (2), and (3) were certainly met except at points either very close to the downlead or within a soil skin depth of the radial ends. For the purpose of the present experiment, condition (4) was regarded as an object of interest, while condition (5) was the main point at issue.

Preliminary computations indicated that there would be sufficient individual wire current to yield usable signal for the instrumentation employed only if relatively few radials made up the ground system. This condition was required also to insure that the signal from a field point over a wire would be large compared to that from the nearby soil current, and moreover to insure that the wire field would not contain significant contribution from adjacent wires. To a radius of $100 \mathrm{ft}$ the soil surface to a depth of several inches was stripped of all old radials, vegetation, and inhomogeneities, and was regraded. Eight No. 30 bare copper wires $40 \mathrm{ft}$ long were laid, stretched, and covered with a thin layer of mud to make contact with the interface as ideal as possible. The inner termination was a 1 -ft-radius brass plate 
grounded to six 18-in. copperweld rods; the outer terminations were $1 / 4$ in. by 6 in. galvanized boat nails. Later, another set of eight radials was run in between the originals. Finally all were extended to $80 \mathrm{ft}$. The antenna was otherwise left as it had been; it is a somewhat conical, 36-radial topload supported at its periphery by a hexagonal spreader stretched over six 8-ft insulated galvanized iron pipe towers symmetrically spaced on a 13 -ft-radius circle; the central support is the $12 \frac{1}{2}-\mathrm{ft}$ downlead. At $1.5 \mathrm{Mc}$, the only frequency employed for both phase and magnitude measurements (an extensive series of magnitude distribution studies was made at 3.0 as well as 1.5), this structure on an ideally conducting ground has a base impedance of $(0.40-j 290)$ ohms, and has an effective height of $7 \frac{1}{2} \mathrm{ft}$.

Figure 2 shows the measurement scheme. It comprised two 1-in.-radius, shielded, 20-turn pickup coils $\mathrm{C}_{1}$ and $\mathrm{C}_{2}$ connected by their coax lines and trimmers $T_{1}$ and $T_{2}$ through similar d-c amplifiers $A_{1}$ and $A_{2}$ to the deflection system of an oscilloscope. Coil $\mathrm{C}_{1}$ was kept at the reference point $1 \mathrm{ft}$ from the downlead over a radial wire, while $\mathrm{C}_{2}$ was the exploring coil. Both coils were oriented for maximum coupling, and the shields of both were insulated from direct contact with soil or wires. An RC phase shifter could be introduced into the circuit of one of the coils in order to sense the absolute direction of an observed phase shift. Base current was monitored by a calibrated milliammeter. The sensitivity of the field-detection system was obtained by comparison with readings on a field-strength meter ( $\mathrm{f}-\mathrm{s}$ meter No. 1) set up beyond the radials, and also by measurement of the induction field of a known, long, straight current filament. Harmonic content of the antenna current was checked as a part of the calibration procedure.

Since the distance $d$ from wire to field point was of the same order as the loop diameter $(d=1.94$ in.) the field cannot be considered uniform over the coil area. The average field $h_{w}$ actually measured is related to the value at the field point by a factor

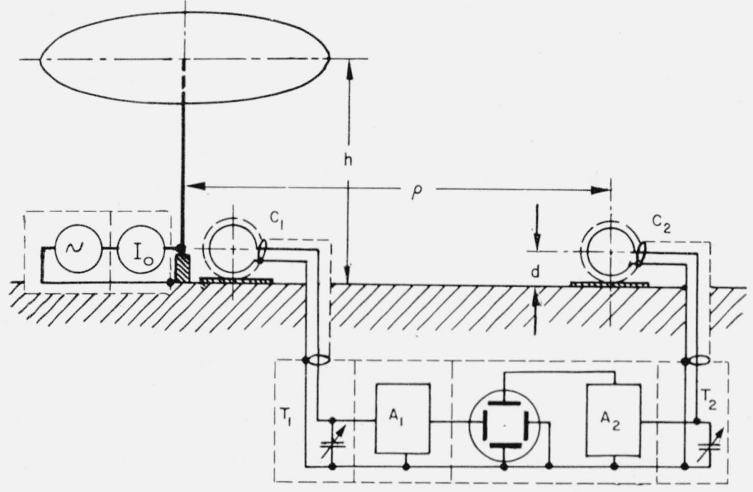

Figure 2. Scheme for measuring field magnitudes and phases.
$h_{w}=F h_{w}$ where for coplanar radial and coil turns

$$
F=\frac{b^{2} \sqrt{1-b^{2} / d^{2}}}{2 d^{2}\left(1-\sqrt{1-b^{2} / d^{2}}\right)} .
$$

For the coils used this amounted to 0.80 . Calibration against f-s meter No. 1 gives $h_{w}=K S_{w} / I_{0}$, where $S_{w}$ is the deflection developed on the oscilloscope, and $K$ is the calibration figure. Stability of $K$ turned out to be to within a percent over an extended period.

For measuring the field between the wires, small trenches were dug and the coil centers alined in the plane of the wires within one-fourth in. These measurements were done largely along the bisectors of the angle between adjacent wires, but exploration was also carried out azimuthally for several fixed values of $\rho$ to test for constancy of field between wires.

The test of equivalence of $H_{\phi}$ and $H_{\phi}^{\infty}$ was a comparison between the near-zone field measured for the antenna over the brass center plate only and the values computed from Wait's formula [12]

$H_{\phi}^{\infty}=\frac{I_{0} e^{-i \beta \rho}}{2 \pi \rho}\left[\left(\frac{h^{2}}{h^{2}+\rho^{2}}\right)^{1 / 2}+i \beta \rho \tan ^{-1}(h / \rho)\right]+D$

where $D$ is the contribution to $H$ from the topload disk, and $h$ is the height of the disk above the ground (also taken to be the effective height). This was done using an untuned probe with a second fieldstrength meter calibrated against f-s meter No. 1. Later a partial confirmation was had from a toploaded monopole set up over a small ground system half of which was hardware-cloth mesh, the other half consisting of 25 radials.

Finally, it was necessary to know something about the electrical characteristics of the soil. To determine these, the properties of short, known lengths of buried open-wire transmission lines of several spacings were measured at the frequencies of interest. From this information the electrical constants of the medium were derived; the best values for 1.5 Mc appeared to be conductivity $\sigma=0.010 \mathrm{mho} / \mathrm{m}$, dielectric constant $k=50$.

\section{Discussion of Results}

Figure 3 shows the results for the test of assumption (4). In general, the agreement between the measured $h_{\phi}$ and theoretical $h_{\phi}^{\infty}$ is good. The departure within the periphery of the topload is not unexpected inasmuch as (a) the topload was somewhat conical rather than flat as assumed in computing $h_{\phi}^{\infty}$, and (b) the soil constants imply a skin depth of about $12 \mathrm{ft}$ at $1.5 \mathrm{Mc}$, so that at no point under the topload can the currents be regarded as flowing in a plane, radial sheet without vertical divergence, which has the effect of decreasing the apparent field. The increase in $h_{\phi}$ over the theoretical curve beyond the topload seems to be mostly due to an 8 percent calibration error discovered in 


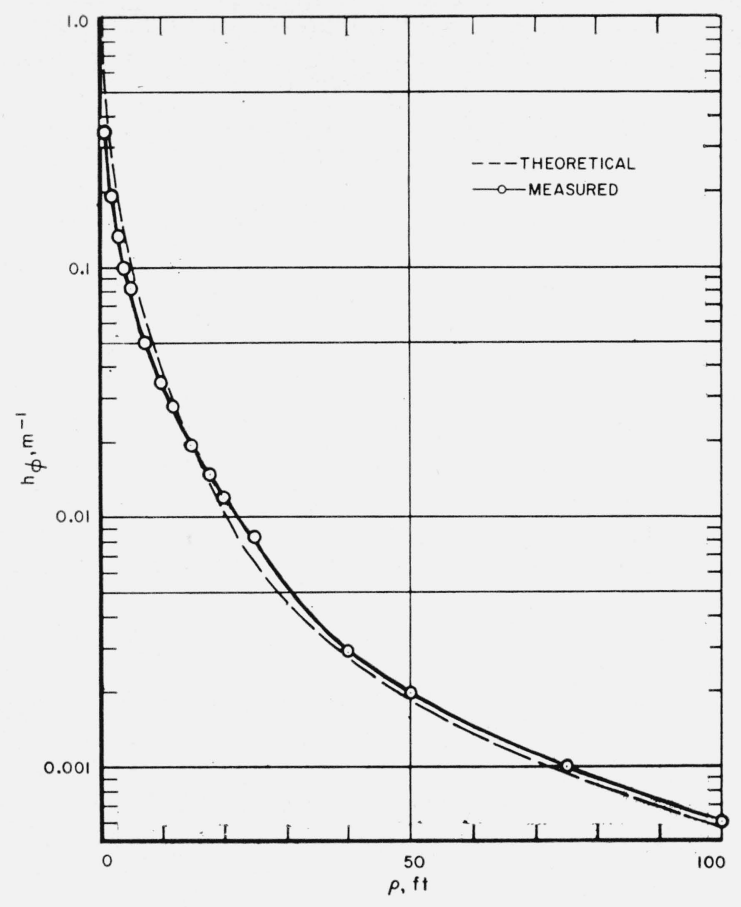

Figure 3. Comparison of measured and theoretical $\mathrm{h} \phi$ for homogenous, poorly conducting ground.

f-s meter No. 1 after the conclusion of the experimental program. Thus for soils no poorer, relatively speaking, than that in this experiment, confirmation seems to be had of the propriety of replacing $h_{\phi}$ by $h_{\phi}^{\infty}$ in $H$-field power loss computations for the region beyond the ground system.

Analysis of the radial ground system experiments proceeds from the observation that there seems to be only qualitative agreement between theory and experiment, and a significant difference between phenomena in the $40-\mathrm{ft}$ system and those in the $80-\mathrm{ft}$ (see figs. 4, 5, and 6). It is first in order to determine the degree to which these somewhat sketchy ground systems satisfy the assumptions of the theory.

Since $\rho H$ changes at most by a factor of two in the ring $\delta \leq \rho \leq 2 \delta$ ( $\delta$ being the skin depth) and less rapidly thereafter, the first assumption appears to be met fairly well except for the area under the topload. The second assumption also appears to be satisfied for the same range of $\rho$ for the 16-radial ground system, but not for the eight. The third assumption was not satisfied by the $80-\mathrm{ft}$ 16-radial system for $\rho>40 \mathrm{ft}$; only poorly in $20 \mathrm{ft}<\rho<40 \mathrm{ft}$, for both $40-$ and $80-\mathrm{ft}$ systems; fairly well in $\rho<$ $20 \mathrm{ft}$. In the eight-radial system it was not satisfied. Assumption (4) seemed to be well satisfied not only from the fact that at $1.5 \mathrm{Mc}|\gamma|=10 \beta$, but also from the experimental confirmation discussed above. Finally, assumption (5) seemed to be only approximately satisfied. Azimuthal sweeps at constant $\rho$ over a quadrant of the ground system showed constancy in $\left|h_{s}\right|$ within a few percent for all locations except those within a horizontal distance of $0.1 \delta$ to

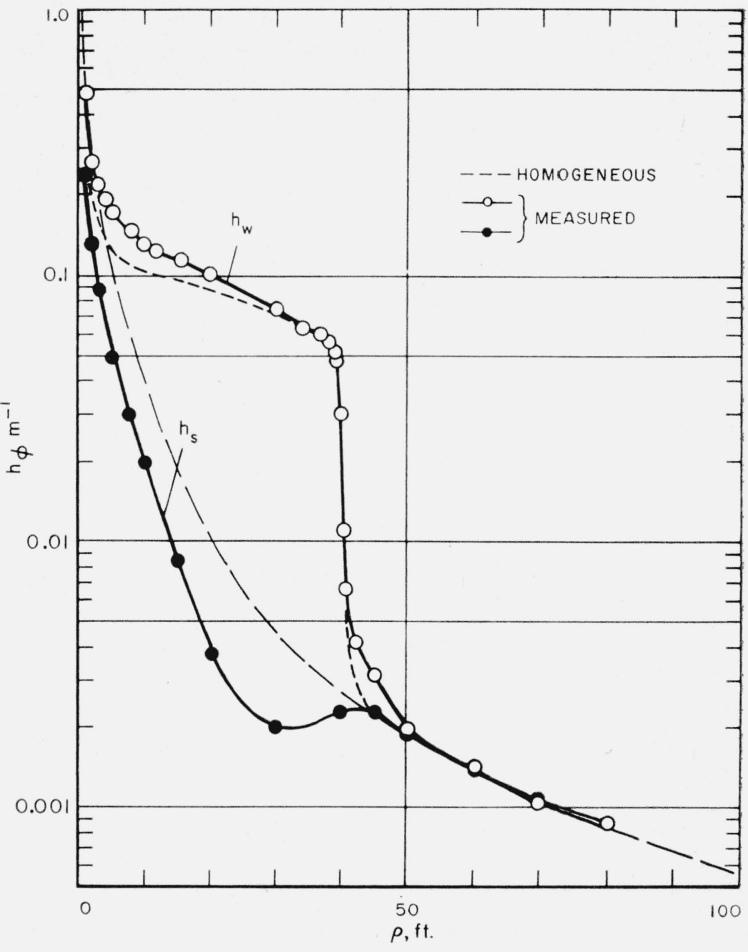

Figure 4. Measured field magnitudes over and between wires of 40-ft 16-radial system.

Short-dashed curve is $\left|h_{w}\right|-\left|h_{\boldsymbol{s}}\right|$, representing minimum possible $h_{w}$.

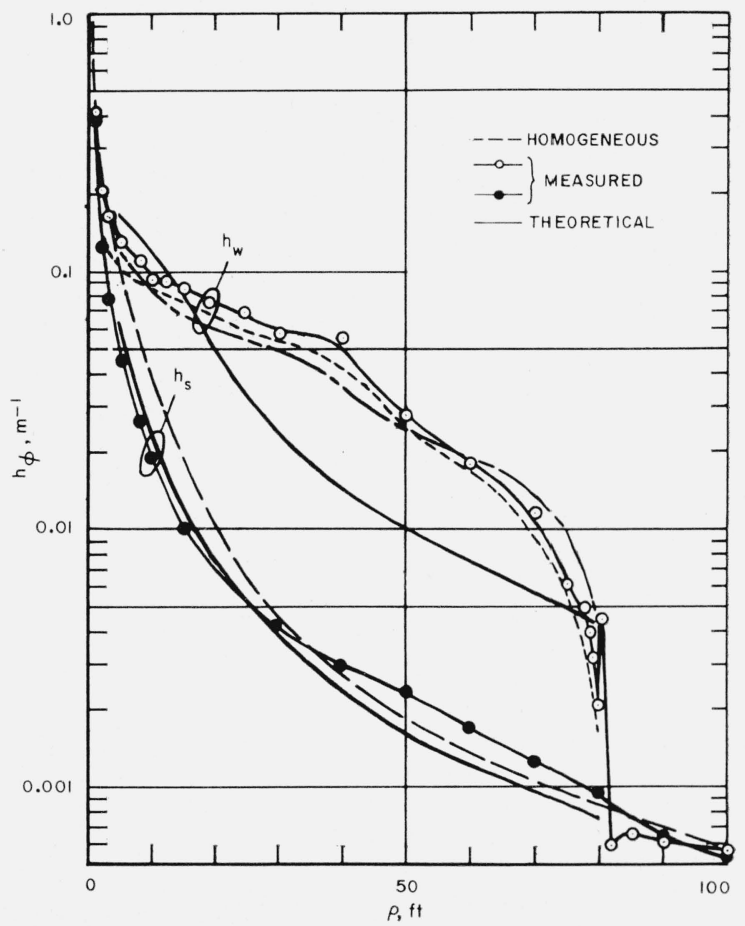

Figure 5. Comparison of measured and theoretical magnitudes over and between wires of 80-ft 16-radial system.

Dash-dot curve is empirical $i_{w}=A e^{\gamma}{ }^{x}+B e-\gamma_{\bullet}{ }^{*}$. 

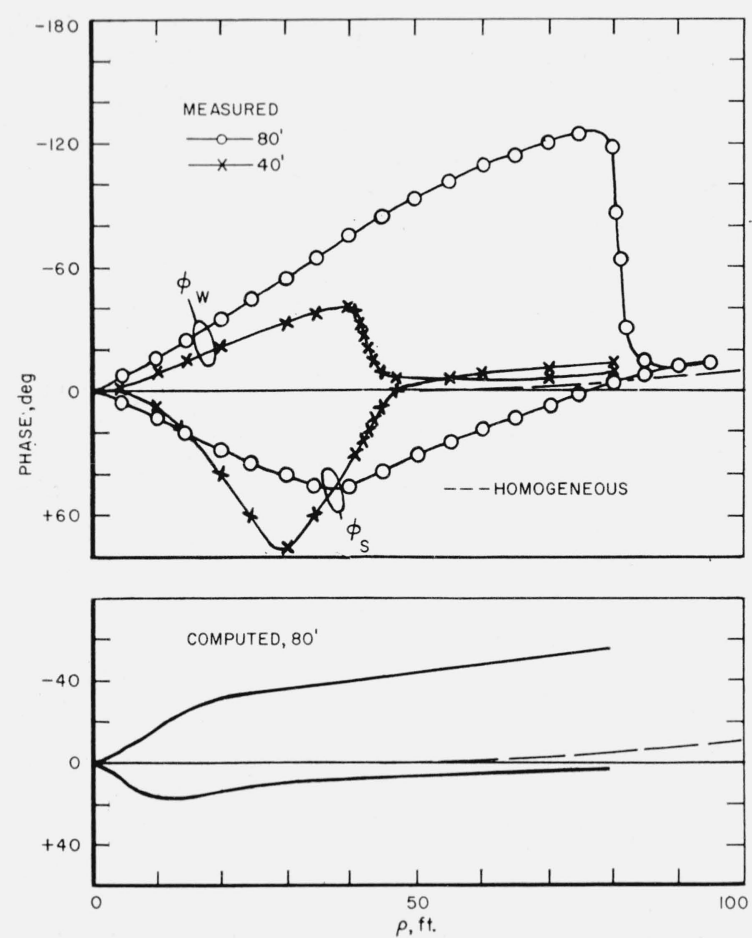

Figure 6. Comparison of measured and theoretical phases for 40- and 80-ft 16-radial ground systems.

Positive phase represents lead relative to downlead current.

the wires, where it showed a decided decrease. This one should expect from the interaction of fields of the parallel-flowing wire and soil currents, but the effect is quite localized.

With reference to the above, then, for $\rho<20 \mathrm{ft}$ measured $\left|h_{s}\right|$ should be expected to fall below the computed values. Figures 4 and 5 show this to be true for both 40- and 80-ft systems. However, for $\rho>20 \mathrm{ft}$, the behavior of $\left|h_{s}\right|$ is distinctly different, the $40-\mathrm{ft}$ system showing reduction in $\left|h_{s}\right|$ at all points, while the $80-\mathrm{ft}$ system shows an enhancement. In the 40-ft system, the wire field in the first $10 \mathrm{ft}$ is very nearly that predicted by current division according to parallel impedances, while for the $80-\mathrm{ft}$ system agreement is only fair. In both systems, beyond $20 \mathrm{ft}$ an increasing departure between measured and computed $\left|h_{w}\right|$ is evident, the nature of which appears different for the two situations.

It is tempting to say simply that, since beyond $20 \mathrm{ft}$ the spacing $s$ between the wires violates the condition $s<<\left|\gamma_{e}^{-1}\right|$, they are acting no longer as a grid but as a collection of $N$ independent, parallel, current filaments, each of whose internal impedance $Z_{i}=R_{i}+j X_{i}$ is given by the usual formulas [13]. Such a radial wire mesh would have at a distance $\rho$ from the common center an equivalent surface impedance of $Z_{w}=2 \pi \rho Z_{i} / N$. At $40 \mathrm{ft}$, the sixteen No. 30 - copper - radial system would by this have $Z_{w}=2.89 / 38.1^{\circ}$. Since the soil impedance in parallel is $Z_{s}=3 \overline{3.1 / 32} .7^{\circ}$ for $\sigma=0.010$ and $k=50$, eq (4) would yield $h_{w}=0.0475 \mathrm{~m}^{-1}$ for this distance; for $\rho=60 \mathrm{ft}, h_{w}=0.022 \mathrm{~m}^{-1}$. Experimentally, one finds in the $40-\mathrm{ft}$ system $h_{w}=0.055$ at $39 \mathrm{ft}$; in the $80-\mathrm{ft}$ system, 0.052 and 0.0183 at 40 and $60 \mathrm{ft}$, respectively. Such agreement seems good until one examines the relation between observed and predicted phases, particularly in the light of the contrasting behavior of $\left|h_{s}\right|$, for the two radial lengths, in the region $\rho>25 \mathrm{ft}$. Thus, it would seem that the $80-\mathrm{ft}$ system acts not merely as a grid of independent wires in parallel with the soil in the outer two thirds.

The nature of the departure of $\left|h_{w}\right|$ from the theoretical values is somewhat suggestive of standing waves. Assuming a transmission-line mode of behavior, one represents the current $i_{w}$ in the wire at a distance $x$ from some reference point by [14].

$$
i_{w}=A e^{\gamma} e^{\mathrm{x}}+B e^{-\gamma_{\mathrm{e}} \mathrm{x}}
$$

where $\gamma_{e}$ is the effective propagation constant of the medium surrounding the wire, and the ratio $B / A$ is the reflection coefficient. Regarding the outer termination as the origin and looking back toward the downlead as the generator, the empirical fit with the experimental curve illustrated by the dash-dot curve of figure 5 is obtained for $\gamma=0.10+i 0.197$, $A=1, B=-1 / 2$, i.e., a lossy line terminated in an impedance higher than its characteristic impedance. Confirmation is had from the figures for $\sigma$ and $k$ derived from $\gamma_{e}{ }^{2}=\left(\gamma_{0}{ }^{2}+\gamma^{2}\right) / 2$. These are $\gamma=0.0064$ $\mathrm{mho} / \mathrm{m}$, and $k=56.4$; also $\lambda=32 \mathrm{~m}$, and $v=0.16 c$ where $c$ is the velocity of light in a vacuum. The buried-transmission-line measurement gave respectively $0.010,50,30$, and 0.15 , which is a fairly reasonable agreement. Since in actuality the wires were a little below the interface, $\sigma$ and $k$ probably were between the above and the values 0.0033 and 29 , respectively, obtained from $\gamma=\gamma_{e}$ (completely buried wire).

The observed phases seem to fit generally with the above picture. The change should be $180^{\circ}$ in $\lambda / 2=16 \mathrm{~m}$. The measured wire field phase referred to the downlead does not change by $180^{\circ}$ in this distance, but advances more slowly at the rate of $1.90^{\circ} / \mathrm{ft}$ instead of $3.40^{\circ} / \mathrm{ft}$. However, if at each point one considers the total phase change of wire field with reference to that at the same radius, the combined change is $1.86^{\circ}+1.29^{\circ}=3.15^{\circ} / \mathrm{ft}$, out to about $50 \mathrm{ft}$ where the total difference is about $130^{\circ}$. The return toward zero for the phase of the field between wires for $\rho>50 \mathrm{ft}$ may be due to masking of the standing-wave circulating-current field in the soil by the incident downlead field. In the case of the $40-\mathrm{ft}$ system, some evidence of the same thing shows up in the phase progression where in the soil it is $2.5^{\circ} / \mathrm{ft}$, in the wire $1.3^{\circ} / \mathrm{ft}$, total $3.8^{\circ} / \mathrm{ft}$, but the magnitude curves, figure 4 , show no recognizable standing wave characteristics. This may be because the region of independently acting radial length is considerably under half a wavelength (for the wire in the interface).

\section{Conclusions}

In both the 16-radial systems as well as in the 8radial, it appears that a substantial potential difference existed between the wires and the soil. Hence, in no case studied experimentally can the grid and 
soil be considered as surface impedances in simple parallel combination. However, in those regions where the ground-system parameters satisfy the remaining four assumptions made in the theory, the observed fields seem to agree with the predicted ones fairly well. In view of the similarity of soil conditions for the present experiment and the previous impedance studies on systems of many radials, it is likely that conclusions drawn here hold for those earlier studies as well.

To compute the $H$-field losses in a radial ground system laid on poorly conducting soil, one properly should consider four regions separately. The inner region is that in which the fields vary more rapidly than the limit specified in the theory; the second (in a proper design, by far the largest) is that in which at least the first four assumptions are satisfied; the third, that in which the wire spacing is such that the radials act independently; the fourth, the terminal region within a soil skin depth of the radial ends. In all four regions, copper losses would be correctly computed only from an observed current distribution, but it seems likely that an adequate approximation would result from using the distribution computed from the parallel impedance assumption; in any reasonable case these losses would be small. The ground losses for the inner region would have to be obtained from a volume current density distribution; they could be (usually are) eliminated by using an effectively solid sheet of highly conducting material in place of the radials. In the second region, where all assumptions but the fifth hold, the losses should be computable from Wait's theory even with very poor contact between wires and soil. The greater the number $N$, the better the results should be for losses computed in this second region. In the third and fourth regions, one has recourse to numerical integration of $\int Z_{s} H_{s}^{2} d A$ where $H_{s}(\rho, 0)$ would have to be an experimentally determined quantity. For very poor soil, poor contact between radials and soil, and mismatched radial termination, sufficient standing-wave circulating current may exist to add a significant extra $H$-field loss component. Under these conditions it would seem worthwhile to direct attention toward providing a termination in the characteristic impedance of the radial regarded as a transmission line.

It must be remembered that the above-described conditions are also those for which considerable charging current power loss can appear in the contact impedance between radials and soil, as well as in the displacement flux terminating in the soil between radials, and in fact such "E-field" losses can be the dominating term in the total ground system loss picture, particularly where the loss tangent of the soil is of the order of unity. For better soils, these losses become of less importance, along with the standing-wave phenomenon, until for $\sigma / \omega k \epsilon_{0}>>10$ they likely are of little significance.
The authors express their appreciation to Harold Hanna and Anthony Muschbacher for their part in the construction, maintenance, and operation of the experimental facility under often very unfavorable climatic conditions, and to James R. Wait of NBS, Boulder Laboratories, for his many helpful comments and continued interest in the project.

\section{References}

[1] J. R. Wait and W. J. Surtees, Impedance of a top-loaded antenna of arbitrary length over a circular grounded system, J. Appl. Phys. 25, 553 (1954).

[2] J. R. Wait and W. A. Pope, The characteristics of a vertical antenna with a radial-conductor ground system, Appl. Sci. Research B4, 177 (1954).

[3] J. R. Wait and W. A. Pope, Input resistance of l.f. unipole aerials, Wireless Engr 32, 131 (1955).

[4] F. R. Abbott and C. J. Fisher, Design of ground system of radial conductors for a vlf transmitter, USNEL Rept. No. 105 (1949).

[5] H. A. Wheeler, Vlf antenna notebook-ground systemarea losses by vertical electric field (unpublished memo, Wheeler Laboratories, Great Neck, N.Y.).

[6] J. R. Wait, On the calculation of transverse current loss in buried-wire ground systems, Appl. Sci. Research B\%, 81 (1958).

[7] W. E. Gustafson, T. E. Devaney, and A. N. Smith, Model studies on vlf antenna ground system design, USNEL Rept. No. 822 (1957).

[8] T. E. Devaney, W. E. Gustafson, and N. H. Balli, Antenna model studies relating to the design of the North Atlantic vlf transmitting station, USNEL Rept. No. 884 (in preparation).

[9] W. G. Hutton, H. F. Cobb, and C. S. Warner, Navy vlf ground system design-field experimental data, 15 Jan 1957, and supplementary field experimental data 15 April 1957 (unpublished memos, Smith Electronics, Inc., Cleveland, Ohio).

[10] C. E. Smith, E. M. Johnson, W. G. Hutton, and G. F. Leydorf, Navy vif ground system design, final report (submitted by Smith Electronics, Inc. to Development Engineering Corp., Washington, D. C., under prime contract NObsr-71360 of Continental Electronics Manufacturing Co., Dallas, Texas).

[11] J. R. Wait, A study of earth currents near a vlf monopole antenna with a radial wire ground system, Proc. IRE 46, 1539 (1958).

[12] J. R. Wait, Earth currents near a top-loaded monopole antenna, J. Research NBS 62, 247 (1959).

[13] American Institute of Physics handbook, pp. 5-91 and 5-201 (McGraw-Hill Book Co., Inc., New York, N.Y., 1957).

[14] J. C. Slater, Microwave transmission, first ed. p. 24 (McGraw-Hill Book Co., Inc., New York, N.Y., 1942).

San Diego, Calif.

(Paper 63D2-16) 